\section{BUTTERFLY FAUNA OF GOVERNMENT ARTS \& SCIENCE COLLEGE CAMPUS, KOZHIKODE, KERALA}

\author{
Alphonsa Xavier \\ Selection Grade Lecturer, Government Arts \& Science College, \\ Kozhikode, Kerala 673018, India \\ Email: puthusery@sify.com
}

Government Arts \& Science College, located in the heart of Kozhikode District in Kerala State, possesses a botanical garden and a medicinal garden. There are about 250 species of plants present in these gardens, which support a wide variety of butterfly species. A preliminary survey for butterflies was carried out by making daily observations in the morning (from 0800 to $1000 \mathrm{hr}$ ) and evening (from 1500 to $1700 \mathrm{hr}$ ) from June 2004 to May 2005 to document the butterfly diversity in the college campus. Collections were also made using a sweep net and the collected specimens were dry preserved following Arora (1990) and deposited in the museum of Zoology Department. Great care was taken to collect only the minimum number of individual species for identification purposes. This was in view of preserving the species density and diversity. Butterflies were also photographed as and when possible. Identification of species was done using available literature (Antram, 2002; Evans, 1932; Gunathilagaraj et al., 1998; Haribal, 1992; Palot et al., 2003; Kunte, 2000) and with the help of experts. Species classification and scientific names are as per Gunathilagaraj et al. (1998). Butterflies observed were categorized into five groups based on their occurrence during the period of study. Accordingly those species which could be observed $80-100 \%$ of days were categorized as very common (VC), $60-80 \%$ as common (C), 40-60\% as occasional (O), 20-40\% as rare (R) and below $20 \%$ as very rare (VR). Population density of individual species was noted by counting the number actually observed daily in the field along transects passing through the garden.

A total of 32 species of butterflies belonging to 27 genera and 12 subfamilies, distributed over five families were detected from the monitoring sites during the study period, as shown in Table 1. The family Nymphalidae dominated with 20 species followed by Pieridae (4 species), Lycaenidae and Papilionidae (3 species each) and Hesperiidae (2 species).

Species like Ariadne merione, Neptis hylas, Euploea core, Charaxes solon, Talicada nyseus, Captopsilia pomona, Leptosia nina and Eurema hecabe were very common and could be seen on all days during the period of observation. Seven species, viz., Melanitis leda, Orsotrioena medus, Junonia lemonias, J. atlites, Polyura athamas, Pachiliopta aristolochiae and Tirumala limniace were common. J. almana, Parantica aglea, Barbo cinnara and Pachliopta hector were occasional visitors Ariadne ariadne, Euthlia aconthea, Ypthima huebneri, Mycalesis perseus, Hypolimnas misippus, Danaus chrysippus, Discophora lepida, Jamides celeno,
Iambrix salsala, Appias albina and Graphium agamemnon were seen rarely. Two species viz., Y. baldus and Curetis thetis were very rare.

Eventhough, the family Nymphalidae exhibited the maximum species diversity, family Pieridae showed maximum species density. Out of the four species of butterflies observed under Pieridae, three species, viz., C. Pomona, L. nina and E. hecabe occurred in large numbers. Among the members of the family Nympahlidae, E. core, showed the maximum density. Among Papilionidae $P$. aristolochiae though exhibited a moderate density, was much less than that of the already mentioned species. All others occurred in varying numbers. Three species of butterflies recorded from the campus have protected status under the Wildlife Protection Act, 1972. The Great Eggfly, Hypolimnas misippus and the Crimson Rose, Pachiliopta hector are protected under Schedule I Part IV, while the Common Albatross, Appias albina under Schedule II Part II. So far, 322 species of butterflies have been recorded from Kerala (Jaffer Palot et al., 2003; Mani, 1997). It is interesting to note that the Government Arts \& Science College Campus at Calicut, having an area of not more than four hectares had a butterfly fauna of 32 species indicate the rich diversity of butterflies in the area. Also, the occurrence of three protected species in the centre of the city where the effects of atmospheric pollution are high is also a commendable feature.

\section{REFERENCES}

Antram, C.B. (2002). Butterflies of India. A Mittal Publication, New Delhi, 226pp.

Arora, G.S. (1990). Lepidoptera, Collection and Preservation of Animals. Published by Zoological Survey of India, Kolkota, pp. 131137.

Arora, K. (2003). Forest Laws. The Wildlife Protection Act, 1972 as amended by the Wild (Protection) Amendment Act, 2002 (Act 16 of 2003). Published by Professional Book Publishers, New Delhi, 85pp.

Evans, W.H. (1932). Identification of Indian Butterflies. Bombay Natural History Society, Bombay, 454pp.

Gunathilagaraj, K., T.N.A. Perumal, K. Jayaram and M. Ganesh Kumar (1998). Some South Indian Butterflies. Field guide. Published under Project Lifescape, Indian Academy of Sciences, Bangalore, 270pp. Haribal, M. (1992). The butterflies of Sikkim, Himalaya and their natural history. Nataraj Publishers, Dehradun, 217pp.

Palot, J., V.C. Balakrishnan and B. Kambrath (2003). Keralathile Chithrasalabhangal. Malabar Natural History Society, Calicut, Kerala, $195 \mathrm{pp}$.

Kunte, K. (2000). India - A Lifescape Butterflies of Peninsular India. Indian Academy of Sciences, Bangalore, Universities Press, 270pp.

Mani, M.S. (1997). Insects. National Book Trust, India, 162pp.

\section{ACKnowledgments}

The author is grateful to C. Radhakrishnan, Officer in Charge and Dr. Jaffer Palot, Senior Scientist of Zoological Survey of India, Kozhikode, for identifying the specimens. 
Table 1. List of butterflies recorded from the campus of Government Arts \& Science College campus, Kozhikode

\begin{tabular}{|c|c|c|c|c|}
\hline S. No. & Scientific Name & Common Name & $\begin{array}{l}\text { Larval Food Plant } \\
\text { in the G.A.S.C. Campus }\end{array}$ & Abundance \\
\hline \multicolumn{5}{|c|}{ Family: Nymphalidae } \\
\hline \multicolumn{5}{|c|}{ Subfamily: Limenitinae } \\
\hline 1 & Ariadne ariadne Linnaeus & Angled Castor & Rivinus communis & $\mathrm{R}$ \\
\hline 2 & Ariadne merione Cramer & Common Castor & Rivinus communis & VC \\
\hline 3 & Neptis hylas Moore & Common Sailor & Helicteres isora & VC \\
\hline 4 & Euthlia aconthea Cramer & Common Baron & $\begin{array}{l}\text { Mangifera indica } \\
\text { Anacardium occidentale }\end{array}$ & $\mathrm{R}$ \\
\hline \multicolumn{5}{|c|}{ Subfamily: Satyrinae } \\
\hline 5 & Ypthima huebneri Kirby & Common Four Ring & Grasses & $\mathrm{R}$ \\
\hline 6 & Ypthima baldus Fabricus & Common Five Ring & Grasses & VR \\
\hline 7 & Mycalesis perseus Fabricus & Common Bush Brown & Grasses & $\mathrm{R}$ \\
\hline 8 & Orsotrioena medus Fabricus & Nigger & Grasses & $\mathrm{C}$ \\
\hline 9 & Melanitis leda Drury & Common Evening Brown & Hetropogon contortus & $\mathrm{C}$ \\
\hline \multicolumn{5}{|c|}{ Subfamily: Nymphalininae } \\
\hline 10 & Junonia lemonias Linnaeus & Lemon Pansy & $\begin{array}{l}\text { Lepidagathis sp., } \\
\text { Nelsonia canescens }\end{array}$ & $\mathrm{C}$ \\
\hline 11 & J. almana Linnaeus & Peacock Pansy & Barleria sp. Osbeckia sp. & $\mathrm{O}$ \\
\hline 12 & J.atlites Linnaeus & Grey Pansy & Barleria sp. & $\mathrm{C}$ \\
\hline 13 & Hypolimnas misippus Linnaeus* & Great Eggfly & $\begin{array}{l}\text { Portulaca oleracea, } \\
\text { Fleurya interrupta }\end{array}$ & $\mathrm{R}$ \\
\hline \multicolumn{5}{|c|}{ Subfamily: Danainae } \\
\hline 14 & Tirumala limniace Gmelin & Blue Tiger & Asclepias curasavica & $\mathrm{C}$ \\
\hline 15 & Parantica aglea Cramer & Glassy Blue Tiger & $\begin{array}{l}\text { Tylophora carnosa, } \\
\text { Cryptolepis buchanani }\end{array}$ & $\mathrm{O}$ \\
\hline 16 & Danaus chrysippus Linnaeus & Plain or Common Tiger & Calotropis procera & $\mathrm{R}$ \\
\hline 17 & Euploea core Cramer & Common Crow & Ichnocarpus fruitiscens & VC \\
\hline \multicolumn{5}{|c|}{ Subfamily: Charaxinae } \\
\hline 18 & Polyura athamas Drury & Common Nawab & $\begin{array}{l}\text { Acacia catechu, } \\
\text { Delonix regia }\end{array}$ & C \\
\hline 19 & Charaxes solon Fabricus & Black Rajah & Tamarind, Wagatea spicata & VC \\
\hline \multicolumn{5}{|c|}{ Subfamily: Morphinae } \\
\hline 20 & Discophora lepida (Moore) & Southern Duffer & Bamboos & $\mathrm{R}$ \\
\hline \multicolumn{5}{|c|}{ Family Lycaenidae } \\
\hline \multicolumn{5}{|c|}{ Subfamily: Polyommatinae } \\
\hline 21 & Jamides celeno Fabricus & Common Cerulean & Sarasa asoka, Caesalpinia sp. & $\mathrm{R}$ \\
\hline 22 & Talicada nyseus (Guerin) & Red Pierrot & Bryophyllum sp. & VC \\
\hline \multicolumn{5}{|c|}{ Subfamily: Theclinae } \\
\hline 23 & Curetis thetis Drury & Indian Sunbeam & Derris scandens, Xylia sp. & VR \\
\hline \multicolumn{5}{|c|}{ Family Hesperiidae } \\
\hline 24 & $\begin{array}{l}\text { Hesperiinae } \\
\text { lambrix salsala (Moore) }\end{array}$ & Chestnut Bob & Grasses & $\mathrm{R}$ \\
\hline 25 & Barbo cinnara (Wallace) & Rice Swift & Oryza sativa, Cymbopogon sp. & 0 \\
\hline \multicolumn{5}{|c|}{ Family Pieridae } \\
\hline 26 & Appias albina C\&R Felder** & Common Albatross & Drypetes oblongifolia & $\mathrm{R}$ \\
\hline 27 & Leptosia nina Fabricus & Psyche & $\begin{array}{l}\text { Capparis heyneana, Crataeva } \\
\text { Religiosa, Cassia fistula }\end{array}$ & VC \\
\hline \multicolumn{5}{|c|}{ Subfamily: Coliadnae } \\
\hline 28 & Captopsilia pomona Fabricus & Common Emigrant & Cassia sp., Bauhimia sp. & VC \\
\hline 29 & Eurema hecabe Moore & Common Grass Yellow & Different species of grasses & VC \\
\hline \multicolumn{5}{|c|}{$\begin{array}{l}\text { Family: Papilionidae } \\
\text { Subfamily: Papilioninae }\end{array}$} \\
\hline 30 & Graphium agamemnon Felder \& Felder & Tailed Jay & $\begin{array}{l}\text { Anona squamosa, Michelia } \\
\text { champa,Polyalthia longifolia }\end{array}$ & $\mathrm{R}$ \\
\hline 31 & Pachliopta hector Linnaeus* & Crimson Rose & Aristoclochia indica & $\mathrm{O}$ \\
\hline 32 & $P$. aristolochiae Fabricus & Common Rose & $\begin{array}{l}\text { Cinnamomum zefanicum } \\
\text { Listea chinensis }\end{array}$ & $\mathrm{C}$ \\
\hline
\end{tabular}

VC - Very Common; C - Common; O - Occasional; R - Rare; VR - Very Rare

* - indicates species coming under Schedule I Part IV and ** - Schedule II Part II of The Wildlife (Protection) Act, 1972. 